\section{PERFIL SOCIODEMOGRÁFICO FAMILIAR E CLÍNICO DE CRIANÇAS COM CARDIOPATIA CONGÊNITA ATENDIDAS EM UMA INSTITUIÇÃO HOSPITALAR}

\author{
Sociodemographic and clinical profile of children with \\ congenital heart disease assisted at a hospital institution
}

\begin{abstract}
Perfil sociodemográfico familiar y clínico de niños con cardiopatía congénita asistidos en una institución hospitalaria
\end{abstract}

Artigo Original

\section{RESUMO}

Objetivo: Conhecer o perfil sociodemográfico familiar e clínico de crianças com cardiopatia congênita atendidas em uma instituição hospitalar. Métodos: Estudo descritivo, transversal, desenvolvido no ambulatório de cardiologia pediátrica de um hospital em Fortaleza-CE, durante o período de março a agosto de 2012. Contemplou uma amostra não aleatória por conveniência de 80 pais de crianças cardiopatas entre 5 e 12 anos. Obtiveram-se informações a partir de dados primários de prontuário e por questionários, abordando as características sociodemográficas dos pais, dados familiares e condições clínicas das crianças. Resultados: Quanto aos dados sociodemográficos dos pais, 77 (96,2\%) dos acompanhantes eram do sexo feminino, 50 (62,5\%) estavam casados ou em união estável, 39 (48,7\%) tinham entre 10 e 12 anos de estudo, e $69(82,6 \%)$ relataram renda familiar entre 1 e 2 salários mínimos. Na amostra de crianças estudada, $43(53,8 \%)$ eram do sexo feminino. A mediana das idades das crianças foi de 8,4 anos, variando entre 5 e 13 anos. Quanto à idade do diagnóstico, 44 $(55,0 \%)$ ocorreram em idade inferior a 6 meses, independentemente do tipo da cardiopatia. Detectou-se que 19 (23,75\%) tinham parentesco com a cardiopatia. Conclusão: Os pais acompanhantes de crianças com cardiopatia eram predominantemente mães, de baixo nível econômico e com escolaridade satisfatória. Houve predominância do diagnóstico precoce, principalmente nas crianças com cardiopatia do tipo cianótica.

Descritores: Cardiopatias Congênitas; Criança; Perfil de Saúde.

\section{ABSTRACT}

Objective: To characterize the sociodemographic and clinical profile of children with congenital heart disease assisted at a hospital institution. Methods: A descriptive, crosssectional study conducted in the pediatric cardiology outpatient clinic of a hospital in Fortaleza, CE, Brazil, in the period from March to August 2012. It comprised a nonrandom convenience sample of 80 parents of children with heart disease aged 5 to 12 years. Information was obtained from primary data of medical records and through questionnaires covering the parent's sociodemographic characteristics, family data and the children's clinical aspects. Results: Regarding socioeconomic data, 77 (96.2\%) caregivers were female, 50 (62.5\%) were married or in a stable relationship, 39 (48.7\%) had 10-12 years of formal education, and 69 (82.6\%) reported a household income between 1 and 2 minimum wages. In the sample of children studied, 43 (53.8\%) were female. The median age of the children was 8.4 years, ranging from 5-13 years. As to the age at the heart disease diagnosis, $44(55 \%)$ occurred under the age of 6 months, regardless of the type of cardiopathy. It was detected that 19 (23.75\%) were related to the disease. Conclusion: Children's caregivers were predominantly their mothers, with low income and satisfactory schooling. Early diagnosis was predominant, mainly among the children with cyanotic cardiopathy.

Descriptors: Heart Defects, Congenital; Child; Health Profile.
1) Universidade de Fortaleza - UNIFOR Fortaleza (CE) - Brasil

2) Universidade Federal do Ceará - UFC Fortaleza (CE) - Brasil

Recebido em: 22/03/2013 Revisado em: 21/05/2013 Aceito em: 23/01/2014 


\section{RESUMEN}

Objetivo: Conocer el perfil socio demográfico familiar y clínico de niños con cardiopatía congénita asistidos en una institución hospitalaria. Métodos: Estudio descriptivo, transversal, desarrollado en el ambulatorio de cardiología pediátrica de un hospital de Fortaleza-CE, en el periodo entre marzo y agosto de 2012. Se incluyó una muestra no aleatoria por conveniencia de 80 padres de niños cardiópatas con edad entre los 5 y 12 años. Se obtuvo informaciones a partir de datos primarios del historial clínico y a través de cuestionarios incluyendo las características socio demográficas de los padres, datos familiares y condiciones clínicas de los niños. Resultados: Respecto a los datos socio demográficos de los padres, 77 (96,2\%) de ellos eran del sexo femenino, 50 (62,5\%) eran casados o vivian en unión estable, 39 (48,7\%) habian estudiado durante 10 y 12 años y 69 (82,6\%) relataron una renta familiar entre 1 y 2 ingresos mínimos. En la muestra de niños estudiada, 43 (53,8\%) eran del sexo femenino. La mediana de las edades de los niños fue de 8,4 anos, variando entre los 5 y 13 años. Respecto a la edad del diagnóstico, 44 $(55,0 \%)$ se dieron en edad inferior a 6 meses, independiente del tipo de cardiopatía. Se detecto que 19 (23,75\%) tenían algún familiar con cardiopatía. Conclusión: Los padres que estaban con los niños cardiópatas eran en su mayoría madres con bajo nivel económico y escolaridad satisfactoria. Hubo predominio del diagnostico precoz principalmente en los niños con cardiopatía del tipo cianótica.

Descriptores: Cardiopatías Congénitas; Niño; Perfil de Salud.

\section{INTRODUÇÃO}

São definidas como cardiopatias congênitas todas aquelas anomalias que atingem a estrutura cardíaca no momento do nascimento. Podem ocorrer na parede do coração, nas válvulas ou nos vasos de irrigação sanguínea. As malformações cardíacas congênitas apresentam amplo espectro clínico e compreendem defeitos que evoluem de forma assintomática e sintomática, ocasionando alta taxa de mortalidade. A maioria das anomalias cardíacas é de etiologia desconhecida, porém, vários fatores estão associados à maior incidência, como pré-natal, mãe com idade acima de 40 anos e genéticos ${ }^{(1,2)}$.

As cardiopatias congênitas (CC) acometem o coração e/ou os grandes vasos sanguíneos da criança ainda no desenvolvimento intrauterino, afetando a anatomia e a fisiologia. São divididas entre acianóticas e cianóticas. Dentre as acianóticas, existem as comunicações (interatrial, interventricular e persistência do canal arterial - PCA) e as estenoses de valvas (pulmonar e aórtica). Dentre as cianóticas, existe a tetralogia de Fallot (TF), a transposição das grandes artérias (TGA), a drenagem anômala de veias pulmonares (DAVP), entre outras. Além disso, têm grandes chances de serem corrigidas cirurgicamente, tornando possível a expectativa de vida normal ${ }^{(3)}$.

No mundo, $2 \%$ a 3\% dos nascidos vivos têm anomalias congênitas. Nos Estados Unidos da América (EUA), 44,5\% destes foram a óbito no primeiro ano de vida e tiveram algum tipo de cardiopatia congênita (CC). Na América Latina, os defeitos cardíacos congênitos são a segunda maior causa de morte em crianças menores de um ano, tornando-se um significativo problema de saúde pública ${ }^{(4)}$.

O avanço da tecnologia aumentou a sobrevida das crianças portadoras de cardiopatia congênita, contudo, o atendimento das necessidades (alimentação, atividade física, cuidado à saúde bucal etc.) é um tópico que merece atenção. As anomalias cardíacas congênitas têm apresentação variada e podem progredir sem sintomas associados, até os relacionados à sintomatologia e à alta mortalidade, traduzindo a variedade e os graus de comprometimento estrutural cardiovascular observados morfologicamente ${ }^{(5,6)}$.

As malformações cardíacas congênitas representam um grupo heterogêneo de doenças com consequências hemodinâmicas variáveis e diferentes necessidades de seguimento e intervenção. A perspectiva do paciente cardiopata e seus interesses podem divergir da opinião dos profissionais da saúde no que concerne a atividades físicas, visitas regulares ao médico, uso de medicamentos, dentre $\operatorname{outros}^{(7,8)}$.

A promoção da saúde (PS) enfatiza, entre suas ações, identificar prioridades de saúde e estabelecer políticas públicas para sua implementação, desenvolver pesquisas que ampliem o conhecimento sobre as áreas prioritárias e implementação dos planos de ação voltados para a qualidade de vida $(\mathrm{QV})^{(9)}$. Nas cardiopatias congênitas, a promoção da saúde compreende a valorização de aspectos diversos, como alimentação, apoio social, responsabilidade sanitária, valorização da vida, exercícios físicos, controle do estresse e comportamento global de promoção da saúde, higiene corporal e odontológica.

A identificação, o diagnóstico e o tratamento das cardiopatias congênitas são resultados do trabalho conjunto de uma equipe multidisciplinar, e o grau de comprometimento e participação de cada especialista tem relação direta com a qualidade do resultado final obtido. Assim, o conhecimento sobre o perfil da população com a qual se trabalha pode apoiar profissionais na elaboração de planos de cuidados e intervenções de prevenção e detecção precoce da anormalidade cardíaca.

Diante desse contexto, objetivou-se conhecer o perfil sociodemográfico familiar e clínico de crianças 
com cardiopatia congênita atendidas em uma instituição hospitalar.

\section{MÉTODOS}

Realizou-se estudo de delineamento quantitativo, descritivo e transversal desenvolvido no ambulatório de cardiologia pediátrica de um hospital de grande porte e de referência para doenças cardiovasculares pediátricas em Fortaleza-CE, Brasil.

Do universo populacional, obteve-se uma amostra não aleatória por conveniência, composta por 80 pais de crianças cardiopatas de 5 a 12 anos, durante o período de março a agosto de 2012.

Para a definição de amostra, utilizaram-se os critérios de seleção nos quais se estabeleceu que as crianças possuíssem diagnóstico médico de $\mathrm{CC}$, independentemente de sexo e do estádio da doença.

Dividiu-se a coleta em duas fases. A primeira, relacionada aos aspectos sociodemográficos familiares. Para essa fase, utilizou-se um questionário aplicado no dia da consulta, padronizado e idealizado pelos pesquisadores e não validado em pesquisa anterior. As variáveis estudadas relacionavam-se ao sexo dos pais acompanhantes, faixa etária (dividida em períodos de cinco anos), situação conjugal, religião, escolaridade (em anos de estudo), renda familiar (em salários mínimos), número de filhos, número de pessoas residentes no domicílio e procedência.

A segunda fase referiu-se às características clínicas da criança. Para a coleta dessas informações, utilizaram-se os dados primários dos prontuários através do registro em um formulário padronizado idealizado pelos pesquisadores e não validado em pesquisa anterior. As variáveis estudadas foram: idade, sexo, tempo e idade do diagnóstico, tipo da $\mathrm{CC}$, antecedentes familiares e parentesco com a cardiopatia.

A análise dos dados consistiu na realização de distribuições de frequências univariadas e medidas descritivas (mediano, mínimo e máximo). Posteriormente, realizaram-se análises bivariadas, estratificadas por tipo de cardiopatia segundo as características clínicas das crianças. Para as variáveis qualitativas nominais (dados clínicos da criança), foram aplicados os testes Qui-Quadrado de Pearson e o Exato de Fischer (frequências $<5$ ). Para as variáveis qualitativas ordinais, os testes Qui-Quadrado de Tendência Linear, a fim de verificar tendências de aumento ou redução entre as proporções e a característica observada (idade do diagnóstico). Para isso, utilizou-se o programa estatístico STATA versão 8, adotando nível de significância de $5 \%$.

A pesquisa, em sua realização, respeitou os preceitos éticos em pesquisa com seres humanos, segundo a
Resolução 466/12 do Conselho Nacional de Saúde. Todos os pais assinaram o TCLE. Foi aprovada pelo Comitê de Ética (Coética) da Universidade de Fortaleza - Unifor (parecer $n^{\circ} 430 / 2011$ ) e pelo Comitê de Ética do hospital onde se realizou a pesquisa (parecer $\mathrm{n}^{\circ} 868 / 2012$ ).

\section{RESULTADOS}

$\mathrm{Na}$ amostra de 80 pais acompanhantes das crianças estudadas, no que se refere às características sociodemográficas, $77(96,2 \%)$ eram mães. A idade do acompanhante variou de 20 a 61 anos, sendo a mediana de 33,7 anos. O grupo etário predominante ficou situado entre 30 e 34 anos, com $25(31,2 \%)$ participantes. Quanto à situação conjugal, 50 (62,5\%) eram casados ou estavam em união consensual (Tabela I).

A religião católica esteve predominante em 51 $(71,2 \%)$ sujeitos. Quanto aos anos de estudo, 39 (48,7\%) acompanhantes possuíam de 10 a 12 anos de estudo. Ao se levar em consideração o valor do salário mínimo, o rendimento de 69 (86,2\%) acompanhantes variou entre $\mathrm{R} \$$ 622,00 e R\$1.200,00, ou seja, de 1 a 2 salários mínimos.

Das famílias estudadas, 58 (72,5\%) tinham entre 2 e 4 filhos. Do total, 46 (57,5\%) conviviam em domicílios entre 4 e 5 pessoas. Quanto à procedência da família, 38 (47,5\%) residiam em Fortaleza.

Da amostra total, $43(53,8 \%)$ crianças correspondiam ao sexo feminino e $37(46,2 \%)$, ao sexo masculino. A mediana da idade das crianças foi de 8,4 anos e variou entre 5 e 13 anos.

Os grupos se mostraram proporcionalmente semelhantes quanto à distribuição etária. Quanto à idade do diagnóstico da cardiopatia, a faixa etária menor de 6 meses prevaleceu tanto nas cianóticas ( $\mathrm{n}=26 / 65 \%)$ quanto entre as acianóticas ( $n=18 / 45 \%)$. Houve uma relação inversamente proporcional entre a idade de diagnóstico e a identificação do diagnóstico $(\mathrm{p}=0,04)$.

Quando relacionado o sexo e a classificação da CC, houve maior frequência do sexo feminino com cardiopatias acianóticas ( $\mathrm{n}=23 / 57,5 \%$ ), enquanto no sexo masculino, 20 (50\%) tinham esse tipo. Não foram identificadas diferenças proporcionais significativas entre o tipo da cardiopatia e o sexo da criança $(\mathrm{p}=0,501)$.

Dentre as cardiopatias do tipo acianóticas, houve maior ocorrência de comunicação interventricular em 11 (13,7\%) crianças. Nas cardiopatias cianóticas, a Tetralogia de Fallot foi verificada em $10(13,7 \%)$. Ao relacionar-se tipo com a idade de diagnóstico, verificou-se maior ocorrência destas nas crianças com idade inferior a 6 meses (Tabela III). 
Tabela I - Características sociodemográficas dos acompanhantes de crianças com cardiopatias (n=80). Fortaleza-CE, 2012.

\begin{tabular}{|c|c|}
\hline Características & n (\%) \\
\hline \multicolumn{2}{|l|}{ Parentesco do acompanhante } \\
\hline Pai & $3(3,7)$ \\
\hline Mãe & $77(96,2)$ \\
\hline \multicolumn{2}{|c|}{ Grupo etário do acompanhante (em anos) } \\
\hline 20 a 24 & $7(8,7)$ \\
\hline 25 a 29 & $19(23,7)$ \\
\hline 30 a 34 & $25(31,2)$ \\
\hline 35 a 39 & $14(17,5)$ \\
\hline 40 a 44 & $7(8,7)$ \\
\hline 45 anos e mais & $8(10)$ \\
\hline \multicolumn{2}{|l|}{ Situação conjugal dos pais } \\
\hline Unidos & $50(62,5)$ \\
\hline Separados & $30(37,5)$ \\
\hline \multicolumn{2}{|l|}{ Religião } \\
\hline Católica & $57(71,2)$ \\
\hline Evangélica & $21(26,2)$ \\
\hline Protestante & $1(1,2)$ \\
\hline Sem religião & $1(1,2)$ \\
\hline \multicolumn{2}{|l|}{ Escolaridade (em anos de estudo) } \\
\hline Até 4 & $1(1,2)$ \\
\hline 5 a 9 & $2(2,5)$ \\
\hline 10 a 12 & $39(48,7)$ \\
\hline 14 ou mais & $38(47,5)$ \\
\hline \multicolumn{2}{|c|}{ Rendimento familiara (em salários mínimos) } \\
\hline$<1$ & $5(6,2)$ \\
\hline 1 a 2 & $69(86,2)$ \\
\hline Superior a 2 & $6(7,5)$ \\
\hline \multicolumn{2}{|l|}{ Número de filhos } \\
\hline 1 filho & $19(23,7)$ \\
\hline 2 a 4 filhos & $58(72,5)$ \\
\hline 5 filhos ou mais & $3(3,7)$ \\
\hline \multicolumn{2}{|c|}{ Número de pessoas que residem no domicílio } \\
\hline Até 3 pessoas & $26(32,5)$ \\
\hline 4 a 5 pessoas & $46(57,5)$ \\
\hline 6 pessoas ou mais & $8(10)$ \\
\hline \multicolumn{2}{|l|}{ Procedência da família } \\
\hline Fortaleza & $38(47,5)$ \\
\hline Interior do Ceará & $29(36,2)$ \\
\hline Outros estados & $5(6,2)$ \\
\hline Litoral e região metropolitana & $8(10)$ \\
\hline
\end{tabular}

Nota: ${ }^{a}$ Salário mínimo (SM) adotado na pesquisa: R\$ 622,00. 
Tabela II - Características clínicas das crianças por tipo de cardiopatia (n=80). Fortaleza-CE, 2012.

\begin{tabular}{|c|c|c|c|}
\hline \multirow[b]{2}{*}{ Característica da criança } & \multicolumn{2}{|c|}{ Cardiopatia } & \multirow[b]{2}{*}{$p$} \\
\hline & $\begin{array}{c}\text { Cianótica } \\
(\mathbf{n}=40) \\
\text { n (\%) }\end{array}$ & $\begin{array}{c}\text { Acianótica } \\
(\mathrm{n}=40) \\
\mathrm{n}(\%)\end{array}$ & \\
\hline $\begin{array}{l}\text { Sexo } \\
\text { Masculino } \\
\text { Feminino }\end{array}$ & $\begin{array}{l}20(50,0) \\
20(50,0)\end{array}$ & $\begin{array}{l}17(42,5) \\
23(57,5)\end{array}$ & $0,501^{\mathrm{a}}$ \\
\hline \multicolumn{4}{|l|}{ Grupo etário (em anos) } \\
\hline $\begin{array}{l}5 \\
6 \text { a } 9 \\
10 \text { ou mais }\end{array}$ & $\begin{array}{c}8(20,0) \\
15(37,5) \\
17(42,5)\end{array}$ & $\begin{array}{c}6(15,0) \\
21(52,5) \\
13(32,5)\end{array}$ & $0,403^{\mathrm{a}}$ \\
\hline \multicolumn{4}{|c|}{ Idade de diagnóstico da cardiopatia } \\
\hline $\begin{array}{l}\text { Menor que } 6 \text { meses } \\
\text { entre } 6 \text { meses e } 1 \text { ano } \\
\text { entre } 1 \text { e } 3 \text { anos } \\
\text { maior que } 3 \text { anos }\end{array}$ & $\begin{array}{l}26(65,0) \\
6(15,0) \\
4(10,0) \\
4(10,0)\end{array}$ & $\begin{array}{l}18(45,0) \\
4(10,0) \\
9(22,5) \\
9(22,5)\end{array}$ & $0,04^{b}$ \\
\hline \multicolumn{4}{|c|}{ Antecedentes familiares com a cardiopatia na família } \\
\hline $\begin{array}{l}\text { Sim } \\
\text { Não }\end{array}$ & $\begin{array}{l}12(30,0) \\
28(70,0)\end{array}$ & $\begin{array}{c}7(17,5) \\
33(82,5)\end{array}$ & $0,189^{\mathrm{a}}$ \\
\hline \multicolumn{4}{|c|}{ Parentesco com a cardiopatia $(n=19)$} \\
\hline $\begin{array}{l}\text { Tios, avós ou bisavós } \\
\text { Filho/Irmão } \\
\text { Cunhado/Sobrinho }\end{array}$ & $\begin{array}{c}7(58,3) \\
4(33,3) \\
1(8,3)\end{array}$ & $\begin{array}{l}4(57,1) \\
1(14,3) \\
2(28,6)\end{array}$ & $0,666^{\mathrm{c}}$ \\
\hline
\end{tabular}

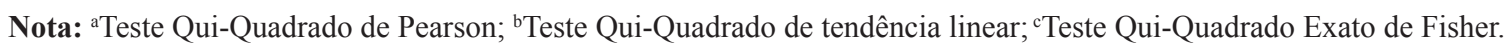

Tabela III - Distribuição percentual das crianças segundo diagnóstico das cardiopatias por idade de diagnóstico. FortalezaCE, 2012.

Idade de Diagnóstico da Cardiopatia

\section{Diagnóstico da Cardiopatia}

\begin{tabular}{|c|c|c|c|}
\hline $\begin{array}{c}\text { Inferior a } 6 \\
\text { meses } \\
\text { n (\%) }\end{array}$ & $\begin{array}{c}\text { Entre } 6 \text { meses } \\
\text { e } 1 \text { ano } \\
\text { n }(\%)\end{array}$ & $\begin{array}{c}\text { Entre } 1 \text { e } 3 \\
\text { anos } \\
\text { n }(\%)\end{array}$ & $\begin{array}{c}\text { Superior a } 3 \\
\text { anos } \\
\text { n (\%) }\end{array}$ \\
\hline $2(4,5)$ & - & & - \\
\hline $2(4,5)$ & - & $1(7,7)$ & - \\
\hline- & - & $1(7,7)$ & - \\
\hline $2(4,5)$ & - & $1(7,7)$ & $2(15,4)$ \\
\hline $5(11,4)$ & $1(10,0)$ & $4(30,8)$ & $2(15,4)$ \\
\hline $2(4,5)$ & - & - & - \\
\hline $1(2,3)$ & - & - & - \\
\hline $6(13,7)$ & $2(20,0)$ & $3(23,0)$ & - \\
\hline $2(4,5)$ & $1(10)$ & - & - \\
\hline- & - & - & $1(7,7)$ \\
\hline $1(2,3)$ & - & - & - \\
\hline $1(2,3)$ & - & - & - \\
\hline $1(2,3)$ & - & - & - \\
\hline- & - & - & $1(7,7)$ \\
\hline $1(2,3)$ & - & - & $1(7,7)$ \\
\hline- & $1(10,0)$ & - & - \\
\hline - & - & - & $1(7,7)$ \\
\hline - & - & - & $2(15,4)$ \\
\hline $7(16,0)$ & $2(20,0)$ & $2(15,4)$ & $1(7,7)$ \\
\hline- & - & - & $1(7,7)$ \\
\hline $1(2,3)$ & - & - & - \\
\hline $1(2,3)$ & - & - & - \\
\hline $6(13,7)$ & $2(20,0)$ & $1(7,7)$ & $1(7,7)$ \\
\hline $3(6,9)$ & $1(10,0)$ & - & - \\
\hline
\end{tabular}




\section{DISCUSSÃO}

A identificação da mulher como provedora de cuidados no contexto familiar de construção sociocultural implica abdicar de muitas coisas, como trabalho, estudo etc., em prol desse papel, particularmente quando o filho apresenta necessidades especiais de saúde. No entanto, a integralidade do cuidado se completa ao inserir o pai nesse contexto, proporcionando maior segurança, sobretudo após a alta hospitalar do filho, o que aumenta o amor e a partilha familiar $^{(11,12)}$.

Sobre a mesma temática, um estudo realizado em Lisboa $^{(11)}$ evidenciou que os pais preocupam-se com o suporte financeiro familiar, não abdicando de suas funções de provedor, enquanto a mãe passa a ser a cuidadora do filho com CC.

No presente estudo, a maioria dos pais era casada ou estava em união consensual. Estudo ${ }^{(13)}$ desenvolvido em Cartagena, Colômbia, com o objetivo de estabelecer a relação entre as características e a qualidade de vida dos cuidadores familiares de crianças com cardiopatias congênitas, evidenciou resultado diferente quanto à situação conjugal, cuja amostra correspondeu a $67 \%$ de pais separados.

A religião católica prevaleceu na atual pesquisa. Pais e familiares de crianças com doenças crônicas têm utilizado a espiritualidade e a religião de diferentes maneiras como mecanismo de enfrentamento. O respeito e o favorecimento das práticas religiosas representam uma forma de apoio e equilíbrio emocional, e podem ser instrumentos significativos para a recuperação das famílias ${ }^{(14)}$. Nesse sentido, a religião é fator relevante no comportamento e evolução da doença nos casos das crianças com cardiopatia, sobretudo porque o aspecto emocional está intrinsecamente relacionado com a sua QV.

Com relação ao tempo de escolaridade dos pais encontrado no presente estudo, de 10 a 12 anos, ele é considerado satisfatório para o bom desempenho no cuidado do filho cardiopata, visto que o conhecimento acerca da patologia poderá ser mais bem compreendido. $\mathrm{O}$ aspecto cultural condiciona ações decisivas na relação entre saúde e doença, revelando os modos de cuidar determinados a partir dos valores e contexto de vida de cada indivíduo ${ }^{(15)}$. Em estudo realizado sobre o conhecimento dos pais acerca da doença de seus filhos com CC, demonstrou-se que há importantes lacunas. Os dados dessa pesquisa sugerem que a forma de abordagem é insuficiente no objetivo de promover uma melhor compreensão dos pais sobre a doença cardíaca da criança e permitir que eles possam transmitir esse conhecimento com precisão aos seus filhos ${ }^{(16)}$.
A renda familiar está relacionada com as oportunidades de um bom desenvolvimento da criança. Segundo um estudo realizado em Fortaleza ${ }^{(15)}$, o qual abordou o impacto do fator econômico sobre o cuidado da criança, na $\mathrm{CC}$, o quadro é mais relevante, visto que o investimento para o cuidado da criança cardiopata torna o orçamento familiar precário, pois impõe a necessidade de renda para garantir o transporte ao hospital, exames de rotina etc. Dessa maneira, verificase a importância de se intensificar o acompanhamento das crianças de baixa renda, por se encontrarem expostas a vários fatores que, combinados, aumentam as chances de desfecho desfavorável ao desenvolvimento da doença ${ }^{(17)}$.

Quanto à idade de diagnóstico da cardiopatia, a faixa etária prevalente na atual pesquisa foi a menor de 6 meses, estando em consonância com a literatura ${ }^{(3)}$, a qual relata que a cardiopatia pode ser identificada ainda no período gestacional, ao nascimento, ou mesmo nos primeiros meses de vida. No entanto, o exame ultrassonográfico obstétrico tem capacidade de detectar apenas as lesões de maior gravidade, sem detecção das de menor repercussão ${ }^{(18)}$.

Uma parcela significativa das crianças investigadas na presente pesquisa possuía cardiopatias múltiplas, com destaque para comunicação interatrial e atresia pulmonar, com $4,5 \%$ em neonatos. Um estudo ${ }^{(6)}$ identificou o total de 357 defeitos cardíacos congênitos, e em 133 pacientes as cardiopatias eram múltiplas, divergindo da presente investigação.

A precocidade das intervenções cirúrgicas no período neonatal justifica-se pela gravidade e complexidade das malformações diagnosticadas e das repercussões hemodinâmicas, com risco de expor o recém-nascido a complicações e morte. Nas cardiopatias complexas e simples, faz-se necessário o procedimento cirúrgico, a fim de controlar os sintomas e melhorar a QV dos pacientes, além de prevenir futuras disfunções.

$\mathrm{Na}$ saúde em geral, mais especificamente na saúde da criança cardiopata, são muitos os obstáculos a serem enfrentados. Traçar o perfil sociodemográfico e clínico dessas crianças torna-se fundamental para que ações sejam implementadas com vistas a um novo paradigma, que não priorize a doença e introduza conceitos facilitadores de promoção da saúde. Portanto, torna-se relevante trabalhar em conjunto com as cinco estratégias de promoção da saúde. A articulação entre esses campos de ação representa uma força maior, impulsionando transformações na realidade de saúde da criança e da família ${ }^{(19,20)}$.

Dessa forma, como as políticas de cuidado em longo prazo depositam na família uma expectativa, devemse buscar soluções para o fornecimento de maior apoio, 
sobretudo social e financeiro, por ser a família fundamental para a excelência dos programas de saúde.

A atividade de cuidar tende a erodir a vida de alguns familiares. Portanto, devem ser realizadas medidas de proteção à saúde do paciente e da família que permitam, por exemplo, planejar e realizar outros interesses que não o cuidado em si.

Como limitação do estudo, encontra-se o número reduzido de crianças com cardiopatias congênitas que participaram no estudo. Acredita-se que são necessários outros estudos, com populações maiores, nos quais se comparem faixas de idade e tipo de cardiopatias.

\section{CONCLUSÃO}

Os pais acompanhantes de crianças com cardiopatia eram predominantemente mães, de baixo nível econômico e com escolaridade satisfatória. Houve predominância do diagnóstico precoce, principalmente nas crianças com cardiopatia do tipo cianótica.

\section{AGRADECIMENTOS}

À Fundação Cearense de Apoio ao Desenvolvimento Científico e Tecnológico - FUNCAP.

\section{REFERÊNCIAS}

1. Rivera IR, Silva MAM, Fernandes JMG, Thomaz ACP, Soriano CFR, Souza MGB de. Cardiopatia congênita no recém-nascido: da solicitação do pediatra à avaliação do cardiologista. Arq Bras Cardiol. 2007;89(1):6-10.

2. Pinto Júnior VC, Daher CV, Sallum FS, Jatene MB, Croti UA. Situação das cirurgias cardíacas congênitas no Brasil. Rev Bras Cir Cardiovasc. 2004;19(2):3-6.

3. Ribeiro C, Madeira AMF. O significado de ser mãe de um filho portador de cardiopatia: um estudo fenomenológico. Rev Esc Enferm USP. 2006;40(1):429.

4. Prieto AM, Massa ER, Torres IEF. Percepción de la calidad de vida de cuidadores de niños com cardiopatia congenita Cartagena, Colombia. Invest Educ Enferm. 2011;29(1):9-18.

5. Damas BGB, Ramos CA, Rezende MA. Necessidade de informação a pais de crianças portadoras de cardiopatia congênita. Rev Bras Crescimento Desenvolv Hum. 2009;19(1):103-13.

6. Leite DL, Miziara H, Veloso M. Malformações cardíacas congênitas em necropsias pediátricas: características, associações e prevalência. Arq Bras Cardiol. 2010;94(3):294-99.

7. Castillo ME, Toro L, Zelada P, Herrera F, Garay R, Alcântara $\mathrm{A}$, et al. Calidad de vida ver pacientes portadores de cardiopatias congênitas. Rev Chil Cardiol. 2010;29(1):57-67.

8. Rosa ET, Trevisan P, Koshiyama DB, Pilla CB, Zen PRG, Varella-Garcia M, et al. Síndrome de deleção 22q11 e cardiopatias congênitas complexas. Rev Assoc Med Bras. 2011;57(1):62-5.

9. Lopes MSV, Saraiva KRO, Fernandes AFC, Ximenes LB. Análise do conceito de promoção da saúde. Texto \& Contexto Enferm. 2010;19(3):461-8.

10. Ministério da Saúde (BR), Conselho Nacional de Saúde. Resolução $n^{\circ} 196$ de 10 de Outubro 1996. Diretrizes e Normas Regulamentadoras de Pesquisa envolvendo Seres Humanos. Brasília: Ministério da Saúde; 1996.

11. Neves TN, Cabral IE. Empoderamento da mulher cuidadora de crianças com necessidades especiais de saúde. Texto \& Contexto Enferm. 2008;17(3):552-60.

12. Simões S, Pires A, Barroca A. Comportamento parental face à cardiopatia congénita. Anál Psicol. 2010;28(4):619-30.

13. Massa ER, Prieto AM, Torres IF. Características de los cuidadores de niños con cardiopatías congénitas complejas y su calidad de vida. Av Enferm. 2010;28(1):39-50.

14. Paula ES, Nascimento LC, Rocha SMM. Religião e espiritualidade: experiência de famílias de crianças com Insuficiência Renal Crônica. Rev Bras Enferm. 2009;62(1):100-6.

15. Frota MA, Albuquerque CM, Linard AG. Educação popular em saúde no cuidado à criança desnutrida. Texto \& Contexto Enferm. 2007;16(2):246-53.

16. Cheuk DK, Wong SM, Choi YP, Chau AK, Cheung YF. Parents' undertanding of their child's congenital heart disease. Heart. 2004;90(4):435-9.

17. Veleda AA, Soares MCF, Cezar-Vaz MR. Fatores associados ao atraso no desenvolvimento em crianças, Rio Grande, Rio Grande do Sul, Brasil. Rev Gaúcha Enferm. 2011;32(1):79-85.

18. Nordon DG, Prigenzi ML. Cardiopatia congênita: difícil diagnóstico diferencial e condução do tratamento. Rev Fac Ciênc Méd Sorocaba. 2012;14(1):24-6.

19. Queiroz MV, Jorge MS. Estratégias de educação em saúde e a qualidade do cuidar e ensinar em pediatria: 
a interação, o vínculo e a confiança no discurso dos profissionais. Interface Comun Saúde Educ. 2006;10(19):117-30.

20. Rocha PA, Soares TC, Farah BF, Friedrich DBC. Promoção da Saúde: a concepção do enfermeiro que atua no programa saúde da família. Rev Bras Promoç Saúde. 2012;25(2):215-20.
Endereço para correspondência:

Mirna Albuquerque Frota

Universidade de Fortaleza

Av. Washington Soares, 1231

Bairro Edson Queiroz

CEP: 60811-905 - Fortaleza - Ceará - Brasil

E-mail: mirnafrota@unifor.br 